\title{
Inhibition of GSK3ß Modulates Cell Death in Epithelial Ovarian Cancer
}

\author{
Naeha Pathak \\ Tyler Walther, BSc \\ Noelle Cutter, PhD \\ Molloy College, Rockville Centre, NY USA
}

doi: 10.19044/esj.2016.v12n15p115 URL:http://dx.doi.org/10.19044/esj.2016.v12n15p115

\begin{abstract}
Epithelial ovarian cancer is one of the most common gynecological malignancies and the fifth most frequent cause of cancer death in women, affecting over 22,000 women annually. Nearly 15,500 affected women die from this disease annually, and chemoresistance from the commonly prescribed platinum-based drug, carboplatin, is a major contributor to this mortality rate. Previous studies have identified genes with CpG islands that are methylated and transcriptionally silenced in resistant epithelial ovarian cancer patients. One of these genes is GSK3 $\beta$, an important regulator of apoptosis and cell growth in the Wnt pathway. Thus, understanding the role of GSK3 $\beta$ suppression in chemoresistance of epithelial ovarian cancer can help contribute to more effective treatments for this disease. By performing assays of cell growth, viability, and apoptosis, our study examined the functional role that GSK3 $\beta$ plays in carboplatin mediated apoptosis. Our results suggest that cells with suppressed GSK3 $\beta$ had increased proliferation and reduced apoptosis. We conclude that silenced GSK3 $\beta$ expression might therefore contribute to carboplatin resistance seen in tumors and our in vitro analysis suggests that GSK3 $\beta$ expression is vital to carboplatin chemosensitivity. Future research is required to further investigate the role of GSK3 $\beta$ methylation to facilitate the design of potential genome-guided treatments for patients with chemoresistant epithelial ovarian cancer.
\end{abstract}

Keywords: Genetics, cancer, methylation, tumor suppressor

\section{Introduction}

Oncologists are constantly seeking new, effective treatments for epithelial ovarian cancer. The primary pharmacological treatment for this disease is a platinum-based drug (typically carboplatin), coupled with a taxane-based drug therapy and surgery (Lum et al., 2013; Marchini et al., 
2012). However, 25\% of these patients present with resistance to the platinum-based drug at diagnosis, and most recurrent tumors ultimately become resistant (Lum et al., 2013; Marchini et al., 2012). The high prevalence of resistant epithelial ovarian tumors warrants the development of new methods to effectively reverse chemoresistance toward carboplatin. Previous studies have shown that several genes, including the gene GSK3 $\beta$ (Glycogen synthase kinase 3 beta), undergo epigenetic changes in epithelial ovarian cancer patients. These changes involve methylation, which causes suppression of gene expression (Koukoura et al., 2014; Lum et al, 2013; Arend et al., 2013). Understanding these epigenetic alterations is important to facilitate the design of new genome-guided therapies (Tang et al., 2015; Nestheide et al., 2013). Therefore, our study investigated the role of GSK3 $\beta$ inhibition in carboplatin chemoresistance of epithelial ovarian cancer cells.

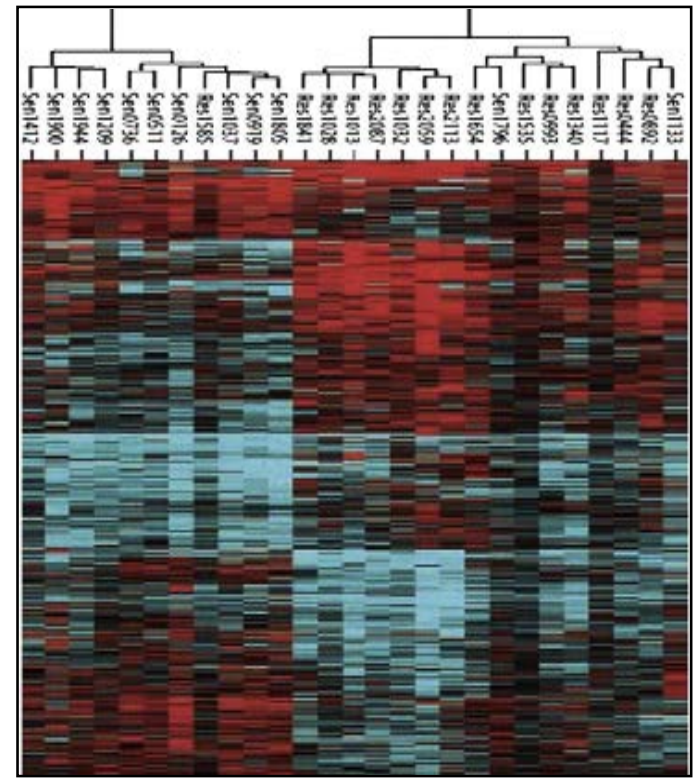

Figure 1: Heatmap of the differentially methylated genes, showing epigenetic segregation between the platinum resistant (Res) and sensitive (Sen) patient groups. Red represents genes that are over-expressed and blue are genes that are under-expressed. Each column represents a patient and each row represents a gene (Lum, 2013).

Epithelial ovarian cancer is one of the most lethal gynecological malignancies in women in the developed world, affecting over 22,000 women annually (Brain et al., 2014). Approximately 9 out of 10 ovarian tumors are classified as epithelial (Brain et al., 2014). Chemoresistance is among the major aforementioned challenges associated with treating this malignancy, thereby requiring further understanding of the underlying molecular mechanisms of this disease. 
Genetic and epigenetic alterations primarily contribute to a tumor's growth, and these alterations can cause resistance to chemotherapeutics (Borley \& Brown, 2015; Liu, Chan, \& Ngan, 2012). Previous studies have identified certain genes that are either under-expressed or over-expressed differentially in chemoresistant and chemosensitive epithelial ovarian cancer patients, as seen in Figure 1 (Borley \& Brown, 2015; Earp \& Cunningham, 2015; Lum et al., 2013). DNA methylation is the transfer of a methyl group to the carbon-5 position of cytosine (Lum et al., 2013). This is an example of an epigenetic alteration, as it regulates the temporal and spatial dynamics of a gene and inhibits its expression (Lum et al., 2013). Recently, DNA methylation has been shown to be involved in signaling pathways that promote tumorigenesis in epithelial ovarian cancer (Borley \& Brown, 2015; Lum et al., 2013). Previous studies have identified CpG islands-areas where cytosine and guanine nucleotides are adjacent to one another-in a family of genes that are methylated and suppressed in patients with carboplatin resistant epithelial ovarian cancer (Koukoura et al., 2014; Lum et al., 2013). Thus, DNA methylation of genes with CpG islands in epithelial ovarian cancer cells can be used as a therapeutic target for developing treatments for this disease.

GSK $3 \beta$, a gene involved in the regulation of Wnt signaling, cell proliferation, and apoptosis (Figure 2), is among the identified genes that is methylated and transcriptionally silenced in chemoresistant epithelial ovarian cancer cells (Lum et al., 2013; Cole, 2013; Jacobs et al., 2012). This gene is an important regulator of apoptosis, a type of genetically programmed cell death that complements cell proliferation in normal tissues (Hilliard et al., 2011). The response of a tumor to a chemotherapeutic agent has been shown to be associated with its apoptotic capacity (Janzen et al., 2015; Han et al., 2013). Thus, GSK3 $\beta$ may contribute to epithelial ovarian cancer cells' response to chemotherapy. In addition, GSK3 $\beta$ plays a major role in the Wnt signaling pathway-a signal transduction mechanism consisting of transmembrane proteins that transmit signals from the extracellular matrix into the cell (Arend et al., 2013; Barbolina, Burkhalter, \& Stack, 2011). As the Wnt pathway is involved in gene transcription, growth, and apoptosis (Arend et al., 2013; Barbolina, Burkhalter, \& Stack, 2011), its associated genes may be implicated in the epigenetics of epithelial ovarian cancer.

The aim of our study was to investigate the effect of GSK3 $\beta$ suppression (mimicking DNA methylation) on carboplatin chemoresistance in epithelial ovarian cancer cells. We hypothesized that the inhibition of GSK3 $\beta$ expression would suppress drug-induced apoptosis and promote proliferation, thereby causing carboplatin chemoresistance. Our in vitro findings may help elucidate the role GSK3 $\beta$ expression plays in chemosensitivity and the relevance of epigenetic regulation to resistant 
epithelial ovarian cancer. Collectively, our results can be used as tools to develop an individualized, genome-guided treatment for epithelial ovarian cancer.

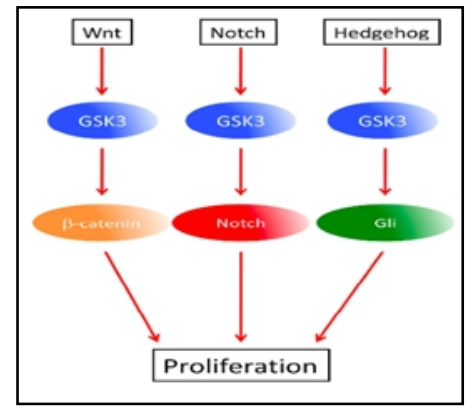

Figure 2: The pathway of GSK3 $\beta$ and its role in regulating cell proliferation, differentiation, and survival (Cole, 2013).

\section{Materials and Methods}

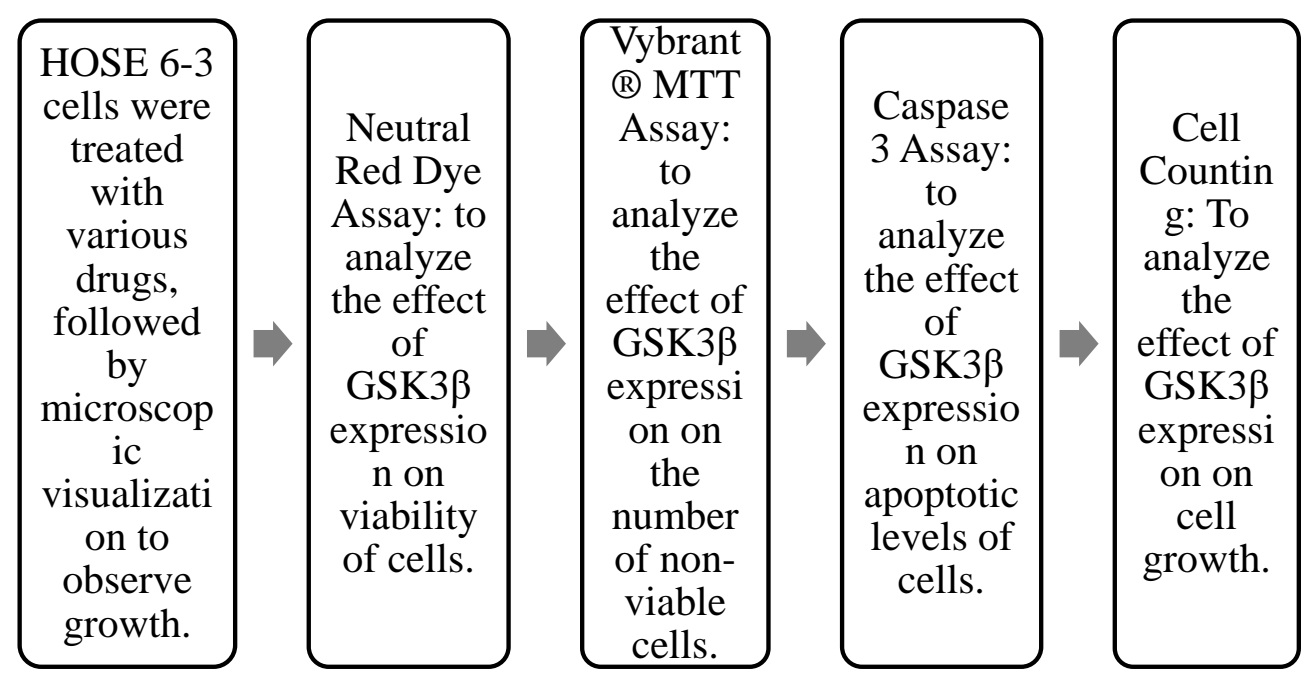

\section{Cell culture and microscopic visualizations}

Human ovarian surface epithelium (HOSE) 6-3 cell line was utilized, which is characterized as sensitive to carboplatin therapy (Lum et al., 2013). The cells were treated with either carboplatin alone (the current platinumbased drug, $200 \mu \mathrm{g} / \mathrm{mL})$ or $\mathrm{LiCl}(2.5 \mathrm{mM})$ followed by carboplatin. $\mathrm{LiCl}$ acts to suppress GSK3 $\beta$ gene expression (Nakada et al., 2011; Grassilli et al., 2013). Throughout the paper, whenever suppression of GSK3 $\beta$ is referred to, this indicates that the cells were pretreated with $\mathrm{LiCl}$. Therefore, cells pretreated with $\mathrm{LiCl}$ did not express GSK3$\beta$, whereas the cells not pretreated expressed GSK $3 \beta$. All cells were supplemented with $10-12 \%$ fetal bovine serum, $2 \%$ penicillin-streptomycin, and grown at $37^{\circ} \mathrm{C}$ with $5 \% \mathrm{CO}_{2}$. We 
took images of cells after two days of incubation using a fluorescence microscope (200X), a fluorescein filter, and a fluorescent dye (Figure 3).

\section{Neutral red dye assay}

We conducted the Neutral Red Dye assay with the "In Vitro Toxicology Assay Kit, Neutral Red Dye Based” (Sigma, Product Code 1002017798) to examine the viability of epithelial ovarian cancer cells. This assay determines the accumulation of the red eurhodin dye (toluylene red) in the lysosomes of viable cells, and thus more absorbance directly correlates with more viability (In vitro toxicology assay kit neutral red based). The cells were studied in six groups: 1) untreated; 2) treated with $\mathrm{LiCl}$ (to suppress GSK3 $\beta$ ); 3) treated with carboplatin; 4) treated with carboplatin+LiCl; 5) treated with doxorubicin (another chemotherapeutic, serving as a comparison); 6) treated with doxorubicin+LiCl. Cells were then split into 96-well plates and incubated for 2 hours with Neutral Red Solution $(10 \mu \mathrm{L})$. Following the protocol, we removed the red dye from the wells and washed the cells with Neutral Red Assay Fixative $(100 \mu \mathrm{L})$. After removing the Neutral Red Assay Fixative, we added the Neutral Red Assay Solubilization Solution $(100 \mu \mathrm{L})$. The 96-well plate was placed on a shaker for ten minutes to complete dissolution. We recorded absorbance at a wavelength of the standard 540nm using the BD Gen 5.1 microplate colorimetric spectrometer.

\section{Vybrant ${ }^{\circledR}$ MTT Cell Assay}

We performed the Vybrant ${ }^{\circledR}$ MTT Cell Assay, using the Vybrant ${ }^{\circledR}$ MTT Cell Assay Kit (ThermoFisher V-13154) to assess the amount of nonviable cells with the treatment groups described in the previous section. The cleavage and conversion of the soluble yellow dye to the insoluble purple formazan is used as a measurement of dead cells (Vybrant ${ }^{\circledR}$ MTT cell assay kit). Following the Vybrant ${ }^{\circledR}$ MTT Cell protocol, a $12 \mathrm{mM}$ MTT stock solution [3-(4,5-dimethylthiazol-2-yl)-2,5-diphenyltetrazolium bromide] was prepared by adding $1 \mathrm{~mL}$ of sterile PBS (phosphate-buffered saline) to a 5 mg vial of MTT. The sample was vortexed until dissolved, $10 \mathrm{~mL}$ of $0.01 \mathrm{M}$ $\mathrm{HCl}$ was added to one tube containing 1 gram of SDS (sodium dodecyl sulfate), and $10 \mu \mathrm{L}$ of the $12 \mathrm{mM}$ MTT stock solution was added to each well. The plate was incubated at $37^{\circ} \mathrm{C}$ for 4 hours. The sample was read at an absorbance of $540 \mathrm{~nm}$ using a spectrometer.

\section{Caspase 3 assay}

We performed the Caspase 3 assay (Abcam, ab39401) using the Caspase 3 Assay Kit to assess the extent of apoptosis in the cells of the aforementioned treatment groups. Following caspase cleavage, a substrate 
for luciferase (aminoluciferin) is released, resulting in a luciferase reaction and the production of light (Caspase 3 assay kit). The absorbance of fluorescent light as measured with the spectrometer directly corresponds to apoptosis (Caspase 3 assay kit). The cells from each sample group were counted using a hemocytometer and microscope; approximately $1.5 \times 10^{6}$ cells were aspirated. Cells were suspended in $50 \mu \mathrm{l}$ of chilled Cell Lysis Buffer and incubated at $5^{\circ} \mathrm{C}$ for ten minutes. Centrifugation at $14,000 \mathrm{rpm}$ was performed for one minute. We then transferred the supernatant into a fresh $1.5 \mathrm{~mL}$ tube. Following the Abcam protocol, we added $50 \mu \mathrm{l}$ of Cell Lysis Buffer, 2X reaction buffer, and DTT in the $1.5 \mathrm{~mL}$ tube. (dithiothreitol) $(10 \mu \mathrm{l})$. The plate was incubated for two hours. Using a spectrophotometer, we measured absorbance at 540nm.

\section{Cell counting}

A cell suspension was prepared in a balanced salt solution (Hanks' Balanced Salts [HBSS], Sigma-Aldrich \# H9269). Samples of HOSE 6-3 cells were either: 1) untreated; 2) treated with carboplatin; 3) treated with carboplatin+LiCl; 4) treated with doxorubicin. On the sixth day, we counted the total number of cells in each sample per $\mu \mathrm{L}$ using a hemocytometer and a light microscope (100X).

\section{Data Analysis}

All data represented in our study is shown as the averages with standard deviation bars of three independent trials per experiment. T-tests were conducted in comparison to the untreated group with Microsoft Excel ${ }^{\circledR}$, with $\mathrm{p}<0.05$ and $\mathrm{p}<0.01$ for significance. All experiments were repeated three times for accuracy, in replicates of 10, unless otherwise stated.

\section{Results}

\section{Cell Cultures and Microscopic Visualizations}

The primary goal of our study was to observe how inactivation of GSK3 $\beta$ contributes to carboplatin resistance in epithelial ovarian cancer cells; thus, we qualitatively observed how GSK3 $\beta$ affects the cells' response to carboplatin. Samples of HOSE 6-3 cells were treated with carboplatin (Figure 1A) and carboplatin+ $\mathrm{LiCl}$ (Figure 2B), independently. Since $\mathrm{LiCl}$ is known to inhibit GSK3 $\beta$ (Grassilli et al., 2013; Nakada et al., 2011), we predicted that the LiCl pretreated cells would show more growth and become resistant to carboplatin. According to Figure 3, more growth was observed in the carboplatin+LiCl group compared to the carboplatin group alone. This observation supports our hypothesis by suggesting that GSK3 $\beta$ expression in carboplatin treated epithelial ovarian cancer cells decreased cell growth and proliferation (Figure 3A). However, GSK3 $\beta$ inhibition increased cell 
growth, suggesting that the cells deficient in expressed GSK3 $\beta$ survived carboplatin treatment (Figure 3B).
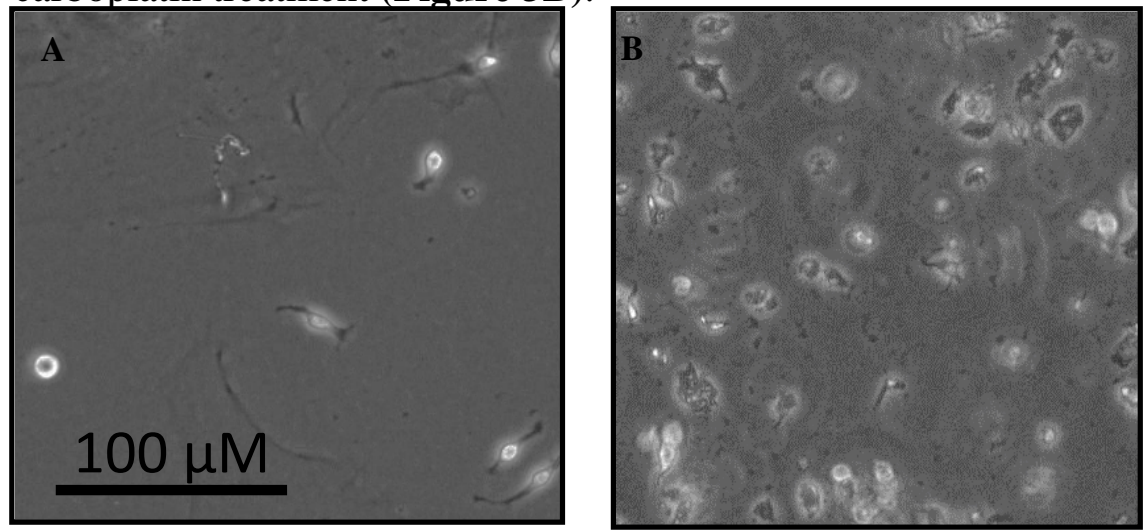

Figure 3: Microscopic visualization (fluorescent microscope, magnified 200X) of HOSE 6-3 cells A) Cells treated with carboplatin exhibited less growth B) Cells treated with carboplatin+ 2.5mM LiCl exhibited more growth

\section{Neutral Red Dye Assay}

We then proceeded to analyze the viability of HOSE 6-3 cells under various treatments with active and silenced GSK3 $\beta$ in order to determine the level of chemoresistance through the Neutral Red Dye assay (Figure 4). Increased absorption of the red dye indicates increased cell viability. HOSE 6-3 cells pretreated with $\mathrm{LiCl}$ were exposed to carboplatin as the main treatment and doxorubicin as an independent control. Another group of cells ( $\mathrm{LiCl}$ untreated) were exposed to carboplatin and doxorubicin (Figure 4). Since $\mathrm{LiCl}$ inhibits GSK3 $\beta$, we predicted that the $\mathrm{LiCl}$ pretreated cells would become resistant to carboplatin (Figure 4). Among the $\mathrm{LiCl}$ untreated cells, those exposed to carboplatin were less viable (absorbance level: $0.38 \mathrm{~nm} \pm 0.13 \mathrm{~nm} \mathrm{SD}, 540 \mathrm{~nm}$, p-value: $1.94 \times 10^{-19}$ ) compared to those left untreated (absorbance level: $1.21 \mathrm{~nm} \pm 0.12 \mathrm{~nm}$ SD, $540 \mathrm{~nm}$ ). However, among the cells pretreated with $\mathrm{LiCl}$, the ones treated with carboplatin showed similar levels of viability (absorbance level: $1.19 \mathrm{~nm} \pm 0.094 \mathrm{~nm}$ SD, $540 \mathrm{~nm}$, p-value: $1.25 \times 10^{-19}$ ) as the untreated ones. Viability of cells treated with doxorubicin remained independent of GSK3 $\beta$ expression. We repeated this process in three independent trials, each consisting of 10 replicates (Figure 4). Each of the three independent trials consistently showed that cells expressing GSK3 $\beta$ with carboplatin treatment were less viable (Figure 4). However, the cells with suppressed GSK3 $\beta$ were more viable upon carboplatin administration, absorbance measured at 540-nm (Figure 4). 


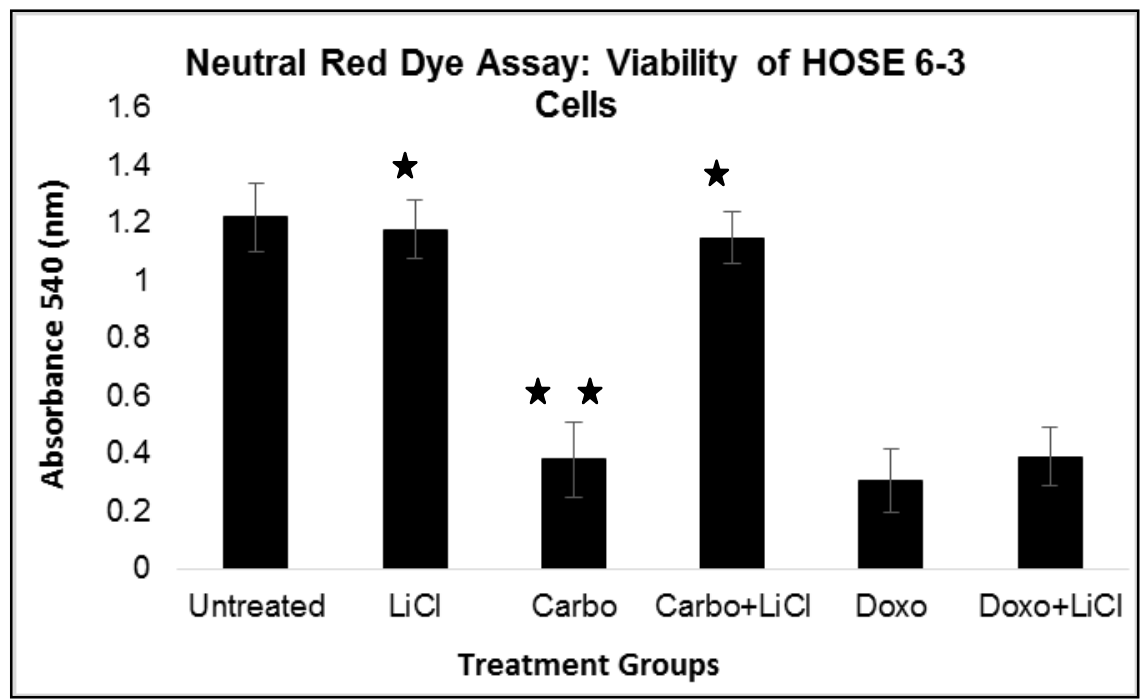

Figure 4: GSK3 $\beta$ determines chemoresistance as a function of viability. Cells treated with carboplatin and doxorubicin had lower viability than cells treated with carboplatin+LiCl. (Carbo=Carboplatin, Doxo=Doxorubicin). The results represent the mean $\pm \mathrm{SD}(\mathrm{n}=3)(*$ denotes $\mathrm{p}<0.05$, ** denotes $\mathrm{p}<0.01$ )

\section{Vybrant ${ }^{\circledR}$ MTT Cell Assay}

To further assess the validity of our hypothesis, we determined whether active GSK3 $\beta$ affects chemoresistance to carboplatin or doxorubicin through the Vybrant ${ }^{\circledR}$ MTT Cell assay. This biochemical assay uses fluorescent indicators indicates greater levels of non-viable cells. Samples of HOSE 6-3 cells were untreated, treated with $\mathrm{LiCl}$ alone, carboplatin, carboplatin $+\mathrm{LiCl}$, doxorubicin (comparison), or doxorubicin $+\mathrm{LiCl}$ (Figure 5). We predicted that the cells with GSK3 $\beta$ suppressed would show greater cell viability compared to the cells with the active form of GSK3 $\beta$. According to Figure 5, the cells treated with carboplatin and doxorubicin (separately) showed the highest absorbance $(1.42 \mathrm{~nm} \pm 0.23 \mathrm{~nm}$ SD and $1.77 \mathrm{~nm} \pm 0.35 \mathrm{~nm} \mathrm{SD}$ respectively, p-values: $3.9 \times 10^{-6}$ and $1.9 \times 10^{-9}$ ), thereby indicating the greatest amounts of non-viable cells. In contrast, the cells treated with carboplatin $+\mathrm{LiCl}$ had similar amounts of cell viability (absorbance level: $0.49 \mathrm{~nm} \pm 0.05 \mathrm{~nm} \mathrm{SD}$, p-values: $3.2 \times 10^{-5}$ ) as the untreated groups (absorbance level: $0.48 \mathrm{~nm} \pm 0.13 \mathrm{~nm} \mathrm{SD}$ ). Each of the three independent trials consistently showed that cells expressing GSK3 $\beta$ with carboplatin treatment were not viable. However, cells with suppressed GSK3 $\beta$ were viable upon carboplatin administration (Figure 5). Additionally, cells treated with doxorubicin $+\mathrm{LiCl}$ had a similar absorbance $\left(1.52 \mathrm{~nm} \pm 0.13 \mathrm{~nm} \quad \mathrm{SD}, \quad \mathrm{p}\right.$-value: $\left.3.4 \times 10^{-2}\right)$ as doxorubicin alone $\left(1.71 \mathrm{~nm} \pm 0.23 \mathrm{~nm}\right.$ SD, p-value: $1.9 \times 10^{-9}$ ) (Figure 5, 540nm absorbance). 


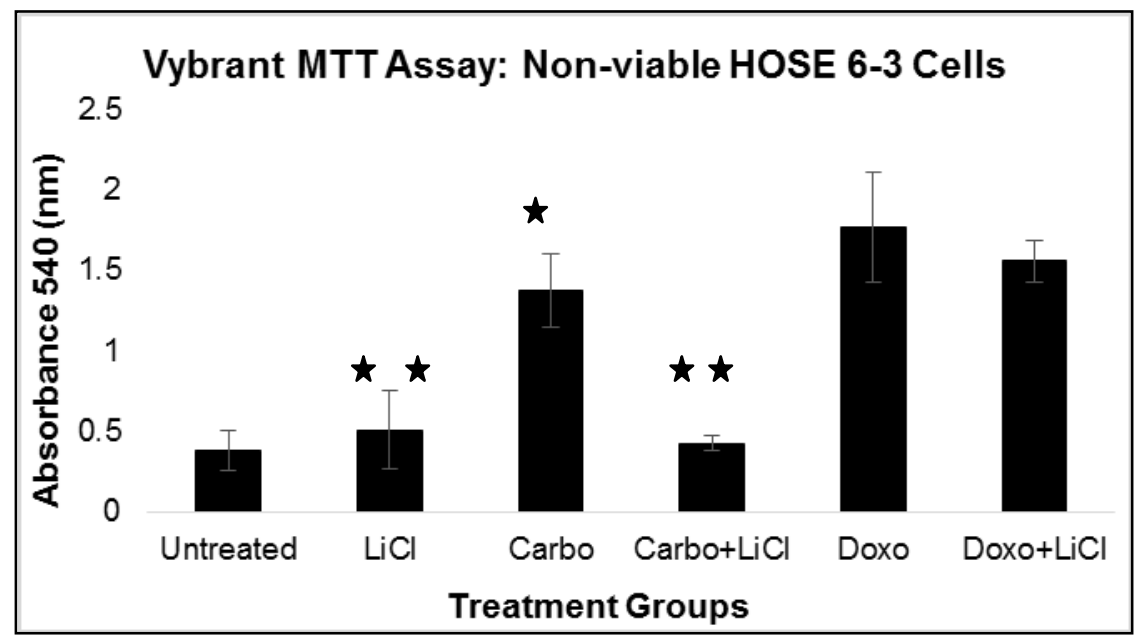

Figure 5: GSK3 $\beta$ determines chemoresistance as a function of non-viability. The sample treated with carboplatin had many non-viable cells. Cells treated with carboplatin and LiCl had a lower amount of non-viable cells, almost identical to that of the untreated cells. (Carbo=Carboplatin, Doxo=Doxorubicin). The results represent the mean $\pm \mathrm{SD}, \mathrm{n}=3$. (* denotes $\mathrm{p}<.05$, ** denotes $\mathrm{p}<.01$ )

\section{Caspase 3 Assay}

GSK3 $\beta$ plays a pivotal role in regulating apoptosis, and since a tumor's response to chemotherapy has been shown to be correlated with apoptotic-induction (Janzen et al., 2015; Han et al., 2013) we sought to analyze apoptosis in cell samples with or without expressed GSK3 $\beta$. To achieve this, we performed the Caspase 3 assay. Treatments mirrored the groups in the previous protocols (Figure 4 and 5). Figure 6 illustrates that the cells treated with carboplatin+LiCl were less apoptotic, compared to cells with carboplatin alone. In other words, cells expressing GSK3 $\beta$ were more susceptible to carboplatin, thus showing increased apoptosis (absorbance levels: $0.69 \mathrm{~nm} \pm 0.12 \mathrm{~nm} \mathrm{SD}$, p-values-7.0 $\times 10^{-8}$ ). This is in direct contrast to cells that did not express GSK3 $\beta$ and were exposed to carboplatin (absorbance level: $0.16 \mathrm{~nm} \pm 0.002 \mathrm{~nm} \mathrm{SD,} \mathrm{p-value:} 1.9 \times 10^{-2}$ ). Figure 6 shows that GSK3 $\beta$ suppression did not affect the extent of apoptosis under doxorubicin treatment but did affect apoptosis under carboplatin treatment. 


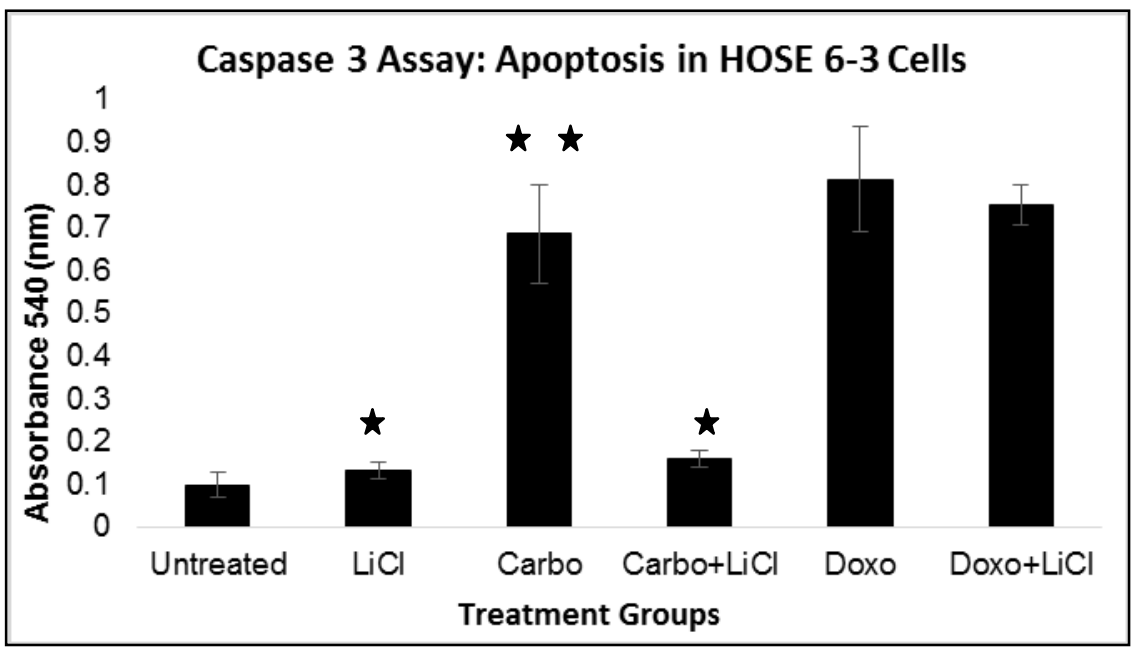

Figure 6: GSK3 $\beta$ determines chemoresistance as a function of apoptosis. Cells treatment with carboplatin had much higher levels of apoptosis than untreated. The cells with carboplatin and $\mathrm{LiCl}$ had very low levels of apoptosis, almost identical to those of the untreated cells. (Carbo $=$ Carboplatin, Doxo=Doxorubicin). The results represent the mean $\pm \mathrm{SD}, \mathrm{n}=3 .{ }^{5}(*$ denotes $\mathrm{p}<0.05, \quad * *$ denotes $\mathrm{p}<0.01)$

\section{Cell Counting}

To quantify cell growth and proliferation of the cells with expressed and suppressed GSK3 $\beta$, we performed cell counts on samples of HOSE 6-3 cells (Figure 7). We had a sample of HOSE 6-3 cells, which were untreated, and another sample that was treated with carboplatin, carboplatin $+\mathrm{LiCl}$, and doxorubicin (independently); these samples were incubated and counted after two days using a hemocytometer and a light microscope (100X). Figure 7 highlights how there was less growth with doxorubicin. Additionally, cells treated with carboplatin+LiCl had similar amounts of growth $\left(1.18 \times 10^{6}\right.$ cells/ $\mu \mathrm{L}, \mathrm{p}$-value: $7.63 \times 10^{-3}$ ) as the untreated cells. All results reflect three independent trials with 10 replicates each. However, cells with carboplatin alone had significantly less growth $\left(6.2 \times 10^{5}\right.$ cells/ $\mu \mathrm{L}$, p-value: $\left.2.93 \times 10^{-2}\right)$. Thus, GSK3 $\beta$ suppression causes more growth following carboplatin exposure. 


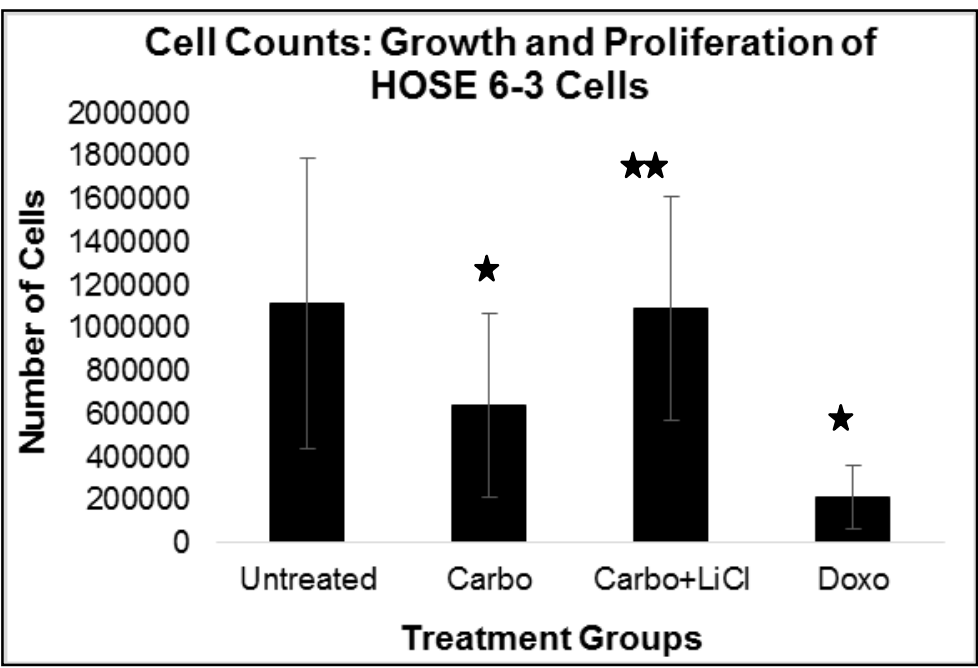

Figure 7: GSK3 $\beta$ determines chemoresistance as a function of cell number. Cell Counting using a light microscope (100X): Cells with carboplatin+LiCl grew and proliferated at almost the same rate as the untreated cells. Cells with carboplatin and doxorubicin grew at a much slower rate. Results represent the mean $\pm S D(n=3)$. (* denotes $\mathrm{p}<0.05$, ** denotes $\mathrm{p}$ $<0.01$ compared to untreated)

\section{Conclusion and Discussion}

Chemoresistance in epithelial ovarian cancer is an important clinical issue as it presents a major limitation to effective treatment and addressing recurrence. Previously, it has been shown that gene expression differs between chemoresistant and chemosensitive epithelial ovarian cancer patients (Figure 1) (Borley \& Brown, 2015; Lum et al., 2013; Cole, 2013). The mechanisms responsible for a tumor's ability to resist treatment are often caused by epigenetic alterations, including gene methylation (Liu, Chan, \& Ngan, 2012). We investigated GSK3 $\beta$ expression to identify potential gene targets for carboplatin resistant epithelial ovarian cancer. GSK3 $\beta$ is a major regulator of cell apoptosis and growth in the Wnt pathway (Lum et al., 2013; Jacobs et al., 2012). This gene has been shown to be methylated and suppressed in epithelial ovarian cancer and has been implicated in chemoresistance of other cancers (Grassilli et al., 2013; Lum et al., 2013; Jacobs et al., 2012). Thus, we examined the role of GSK3 $\beta$ in epithelial ovarian cancer cells that were known to be carboplatin sensitive as described by our previous publication (Lum et al., 2013). In our study, we assessed cell viability, apoptosis and growth (Figures 3 to 7) in the HOSE 63 cell line, pretreated with $\mathrm{LiCl}$ (GSK3 $\beta$ suppressed) or without $\mathrm{LiCl}$ (GSK3 $\beta$ expressed). LiCl inhibits GSK3 $\beta$ expression through two mechanisms: 1) Promoting Ser-9 phosphorylation, an inhibitory marker, and 2) Blocking $\mathrm{Mg}^{2+}$ ions from binding, thus preventing activation of GSK3 $\beta$ 
(Grassilli et al., 2013; Nakada et al., 2011). In the future we plan to further elucidate the mechanisms involved in the signal transduction pathway of GSK3 $\beta$ in our cell lines.

Microscopically visualizing cells under different treatments was necessary in studying the role of GSK3 $\beta$ inhibition in cell growth and carboplatin chemoresistance (Figure 3). There were fewer viable cells after carboplatin exposure in cells without $2.5 \mathrm{mM} \mathrm{LiCl}$ treatment compared to cells with $\mathrm{LiCl}$ treatment; this suggests the role of GSK3 $\beta$ expression in cell protection and apoptosis (Figure 3). Results from the Neutral Red Dye assay, Vybrant ${ }^{\circledR}$ MTT Cell assay, Caspase 3 assay, and direct cell counts assays provided further support for our hypothesis (Figure 4 to 6).

Analyzing viability of the cells under different treatments was critical to studying the role of GSK3 $\beta$ inhibition in chemoresistance. We determined overall cell viability with the Neutral Red Dye and Vybrant ${ }^{\circledR}$ MTT Cell assays. According to the Neutral Red Dye assay, carboplatin treatment significantly decreased cell viability in cells that expressed GSK3 $\beta$ (p-value: $1.94 \times 10^{-19}$ ) (Figure 4). However, in cells with silenced GSK3 $\beta$, carboplatin was not effective in decreasing viability (p-value: $1.25 \times 10^{-19}$ ) (Figure 4). This suggests that GSK3 $\beta$ is critical to cellular viability, and suppressing its expression may result in chemoresistance. Sensitivity to doxorubicin remained independent of GSK3 $\beta$ expression, suggesting that GSK3 $\beta$ inhibition affects carboplatin resistance, but not doxorubicin resistance. This is perhaps because doxorubicin has a different mechanism of action that may be GSK3 $\beta$ independent (Thorn et al., 2012). Future work in our lab continues to examine the variable mechanisms of action of both drugs.

Next, the Vybrant ${ }^{\circledR}$ MTT Cell assay analyzed cell viability with active and silenced GSK3 $\beta$, treated with carboplatin and doxorubicin, independently (Figure 5). Our results show that the untreated cells had almost identical amounts of non-viable cells as those treated with carboplatin+LiCl (p-value: $3.2 \times 10^{-5}$ ); this once again suggests that suppressing GSK3 $\beta$ can potentially lead to carboplatin chemoresistance (Figure 5), as it resulted in a direct effect on cellular viability. However, the HOSE 6-3 cells with expressed GSK3 $\beta$ were significantly less viable upon carboplatin exposure, suggesting that the cells were selectively transformed upon the addition of $\mathrm{LiCl}$ and remained chemosensitive (p-value: $3.9 \times 10^{-6}$ ) (Figure 5). Additionally, the sample treated with doxorubicin and doxorubicin+LiCl independently had similar amounts of non-viable cells, suggesting that GSK3 $\beta$ suppression does not affect resistance to doxorubicin. Future studies are required to elucidate how the suppression of GSK3 $\beta$ results in carboplatin resistance, and how the expression of GSK3 $\beta$ results in carboplatin sensitivity by looking at upstream and downstream targets in the 
Wnt/ $\beta$-catenin pathway. Current research in our lab suggests that GSK3 $\beta$ acts independently in this role (results not shown).

One major path of chemoresistance involves the dysregulation of apoptosis. Dysfunctional apoptotic responses may result in uncontrolled cell growth, leading to cancer (Han et al., 2013). The efficacy of most chemotherapeutics, such as platinum-based drugs, relies heavily on their ability to induce apoptosis (Lum et al., 2013). As a consequence, chemoresistant cells often have down-regulated pro-apoptotic genes or upregulated anti-apoptotic genes (Lum et al., 2013). To determine whether GSK3 $\beta$ expression specifically affects apoptosis, the Caspase 3 assay was performed (Figure 6). Since GSK3 $\beta$ is involved in regulating apoptosis (Lum et al., 2013; Jacobs et al., 2012), we analyzed apoptosis levels in cells with various treatments, with or without GSK3 $\beta$ (Figure 6). Carboplatin induced a significantly greater level of apoptosis in this cell line (p-value: 7.0 $\mathrm{x} 10^{-8}$ ) when compared to untreated cells (Figure 6). These results suggest that the cells with active GSK3 $\beta$ continue to undergo apoptosis when treated with carboplatin. In contrast, cells treated with carboplatin+LiCl (p-value: $1.9 \times 10^{-2}$ ), showed no difference in levels of apoptosis compared to untreated cells (Figure 6), strongly suggesting that GSK3 $\beta$ suppression plays a critical role in modification of carboplatin apoptotic pathways. When GSK3 $\beta$ was inhibited in cells treated with doxorubicin ( $\mathrm{p}$-value: $4.1 \times 10^{-7}$ ), the sample showed nearly identical levels apoptosis as the sample with GSK3 $\beta$ expressed (p-value: $7.6 \times 10^{-5}$ ); this finding suggests that GSK3 $\beta$ affects carboplatin apoptotic pathways selectively; this may be because of the fact that carboplatin and doxorubicin belong to different classes of drugs; carboplatin is classified as an alkylating agent, whereas doxorubicin is an anthracycline antibiotic (Thorn et al., 2012). Thus, GSK3 $\beta$ may regulate carboplatin chemosensitivity because it is associated with the same apoptotic pathway that carboplatin induces. Doxorubicin has a different mechanism of action that we predict is GSK3 $\beta$ independent (Thorn et al., 2012). Figure 6 highlights the expression of GSK3 $\beta$ as a determinant for carboplatin-induced apoptosis among HOSE 6-3 cells. These results demonstrate that the inhibition of GSK3 $\beta$ reduces carboplatin-induced apoptosis, which may be responsible for chemoresistance as observed in clinical cases (Conic et al., 2015; Lum et al., 2013). We have seen the same modification of apoptosis in our lab using cisplatin, another alkylating agent with similar properties to carboplatin, suggesting GSK3 $\beta$ plays a selective role in platinum based apoptotic pathway signaling (results not shown). Platinum based drugs have the ability to add alkyl groups to many electronegative groups, thereby crosslinking strands of DNA (Sharon et al., 2009).

Cell growth was assessed directly through cell count (Figure 7). There was significantly less cell growth with doxorubicin (p-value: $3.89 \mathrm{x}$ 
$10^{-2}$ ). There was less cell growth with carboplatin as well (p-value: $2.93 \times 10^{-}$ $\left.{ }^{2}\right)$. However, there was significantly more cell growth when the sample was pretreated with $\mathrm{LiCl}$ followed by carboplatin (p-value: $7.63 \times 10^{-3}$ ) (Figure 7). Collectively, our results support our other studies (Lum et al., 2013) and demonstrate that suppression of GSK3 $\beta$ leads to modification of cellular viability by increasing cellular growth when GSK3 $\beta$ is silenced. Importantly, our results suggest that this change in cellular viability can result in chemoresistance, and expression of GSK3 $\beta$ leads to carboplatin chemosensitivity.

Epithelial ovarian cancer is one of the deadliest gynecological malignancies that affects women worldwide (Liu, Chan, \& Ngan, 2013). Evidence from previous studies shows that genetic and epigenetic alterations are major underlying mechanisms that contribute to tumorigenesis, especially in epithelial ovarian cancer (Borley \& Brown, 2015; Liu, Chan, \& Ngan, 2013). Chemoresistance to the current platinum-based therapy in such cases is a major obstacle for the clinical management of epithelial ovarian cancer (Borley \& Brown, 2015; Liu, Chan, \& Ngan, 2012). Thus, developing a treatment that prevents or reverses chemoresistance is vital for the survival of epithelial ovarian cancer patients. To overcome this challenge, it is important to study genes involved in epithelial ovarian cancer. Recent studies have identified genes with $\mathrm{CpG}$ islands that undergo epigenetic alterations, particularly methylation, and are associated with the acquisition of chemoresistance in epithelial ovarian cancer (Koukoura et al., 2014; Lum et al., 2013; Zeller et al., 2011). Specifically, GSK3 $\beta$ is a gene with CpG islands that has been shown to be transcriptionally silenced via methylation in resistant epithelial ovarian cancer patients (Lum et al., 2013; Jacobs et al., 2012). Therefore, our study focused on GSK3 $\beta$ and its relation to carboplatin chemoresistance in epithelial ovarian cancer. By performing various assays of cell growth, viability, and apoptosis, our study successfully supported our hypothesis by demonstrating that GSK3 $\beta$ suppression results in tumorigenic properties and carboplatin chemoresistance (Figures 3 to 7). These results strongly suggest that GSK3 $\beta$ expression plays a significant $(p<0.05$ or $p<$ 0.01 ) role in carboplatin sensitivity, supporting previous studies that have been done involving other genes with chemoresistant epithelial ovarian cancer (Lum et al., 2013). In the future, therapies can be developed that target GSK3 $\beta$ and the Wnt pathway. These findings hold promise for future genome-guided chemotherapy based on a patient's individual gene expression profile (Tang et al., 2015; Nestheide et al., 2013).

Another approach we propose is to target other downstream effectors of GSK3 $\beta$ in the Wnt signaling pathway, such as $\beta$-catenin (Figure 2). Currently this is an active area of research in our lab. Additionally, our recent research suggests that blocking methylation with a demethylating 
enzyme can reverse carboplatin chemoresistance (Cheishvili et al., 2015). However, it is difficult to solely block methylation of a single gene in vivo without disrupting other normal epigenetic processes (Handy, Castro, \& Loscalzo, 2012). Hence, we can use our data and explore the Wnt/ $\beta$-catenin pathway to create a more targeted treatment. Figure 2 suggests that inhibiting this gene via methylation would inhibit downstream effectors of GSK3 $\beta$, such as $\beta$-catenin, resulting in uncontrolled proliferation and tumorigenic properties (Arend et al., 2013; Cole, 2013). Thus, we suggest that activating these downstream effectors, such as $\beta$-catenin, would be effective in circumventing this process and blocking proliferation signaling. Additional studies in upregulating $\beta$-catenin are necessary to examine the efficacy of this approach. We plan to actively research this in the future.

Our study demonstrates that the inhibition of GSK3 $\beta$ plays a major role in carboplatin chemoresistance of epithelial ovarian cancer, and its gene expression promotes carboplatin sensitivity. Studying the role of epigenetics and methylated genes, such as GSK3 $\beta$, in chemoresistance of epithelial ovarian cancer can lead to increased accuracy in diagnosis, prognosis, and treatment of this disease. All the above-stated investigations could improve treatment efficacy, reduce the need for more aggressive treatment modalities, and facilitate the development of specific genome-guided therapy that target resistant epithelial ovarian tumors.

\section{References:}

Arend, R., Londoño-Joshi, A., Straughn Jr., M., \& Buchsbaum, D. (2013). The Wnt/ $\beta$-catenin

pathway in ovarian cancer: A review. Gynecologic Oncology, 131(3), 772779.

Barbolina, M. V., Burkhalter, R. J., \& Stack, M. S. (2011). Diverse mechanisms for activation of Wnt signalling in the ovarian tumour microenvironment. Biochemical Journal, 437(1), 1-12.

Borley, J., \& Brown, R. (2015). Epigenetic mechanisms and therapeutic targets of chemotherapy resistance in epithelial ovarian cancer. Annals of Medicine, 47(5), 359-369.

Brain, K. E., Smits, S., Simon, A. E., Forbes, L. J., Roberts, C., Robbé, I. J., \& Hanson, J. (2014). Ovarian cancer symptom awareness and anticipated delayed presentation in a population sample. BMC Cancer, 14(171).

Caspase 3 assay kit (colorimetric). (2012). Retrieved from Abcam website: http://www.abcam.com/ps/products/39/ab39401/documents/ab39401\%20Cas pase\%203\%20Assay\%20Kit\%20Colorimetric\%20(Website).pdf

Cheishvili, D., Boureau, L., Szyf, M. (2015). DNA demethylation and invasive cancer: implications for therapeutics. $\mathrm{Br} J$ Pharmacol, 172(11), 2705-2715. 
Cole, A. R. (2013). Regulation of cell fate in the brain by GSK3. Trends in Cell Signaling Pathways in Neuronal Fate Decision.

Conic, I., Stanojevic, Z., Velickovic, L. J., Stojnev, S., Petrovic, A. R., Krstic, M., \& Stefanovic, V. (2015). Epithelial ovarian cancer with CD117 phenotype is highly aggressive and resistant to chemotherapy. Journal of Obstetrics and Gynaecology Research, 41(9).

Earp, M. A., \& Cunningham, J. M. (2015). DNA methylation changes in epithelial ovarian cancer histotypes. Genomics, 106(4).

Grassilli, E., Narloch, R., Federzoni, E., Ianzano, L., Pisano, F., Giovannoni, R., \& Lavitrano, M. (2013). Inhibition of GSK3B bypass drug resistance of p53-null colon carcinomas by enabling necroptosis in response to chemotherapy. Clinical Cancer Research, 19(14).

Han, Z., Feng, J., Hong, Z., Chen, L., Li, W., Liao, S., \& Gao, Q. (2013). Silencing of the STAT3 signaling pathway reverses the inherent and induced chemoresistance of human ovarian cancer cells. Biochemical and Biophysical Research Communications, 435(2).

Handy, D. E., Castro, R., \& Loscalzo, J. (2011). Epigenetic Modifications: Basic Mechanisms and Role in Cardiovascular Disease. Circulation, 123(19), 2145-2156.

Hilliard, T. S., Gaisina, I. N., Muehlbauer, A. G., Gaisin, A. M., Gallier, F., \& Burdette, J. E. (2011). Glycogen synthase kinase 3 beta inhibitors induce apoptosis in ovarian cancer cells and inhibit in vivo tumor growth. AntiCancer Drugs, 22(10), 978-985.

In vitro toxicology assay kit neutral red based. (n.d.). Retrieved from Sigma website: $\quad$ https://www.sigmaaldrich.com/content/dam/sigmaaldrich/docs/Sigma/Bulletin/tox4bul.pdf

Jacobs, K. M., Bhave, S. R., Ferraro, D. J., Jaboin, J. J., Hallahan, D. E., \& Thotala, D. (2012). GSK-3ß: A Bifunctional Role in Cell Death Pathways.International Journal of Cell Biology, 2012, 930710.

Janzen, D. M., Tiourin, E., Salehi, J. A., Paik, D. Y., Lu, J., Pellegrini, M., \& Memarzadeh, S. (2015). An apoptosis-enhancing drug overcomes platinum resistance in a tumour-initiating subpopulation of ovarian cancer. Nature Communications, 6(1).

Koukoura, O., Spandidos, D. A., Daponte, A., \& Sifakis, S. (2014). DNA methylation profiles in ovarian cancer: Implication in diagnosis and therapy (Review). Molecular Medicine Reports, 10(1), 3-9.

Liu, M. X., Chan, D. W., \& Ngan, H. Y. (2012). Mechanisms of chemoresistance in human ovarian cancer at a glance. Gynecology \& Obstetrics, 2(3).

Lum, E., Vigliotti, M., Banerjee, N., Cutter, N., Wrzeszczynski, K. O., Khan, S., \& Lucito, R. 
(2013). Loss of DOK2 induces carboplatin resistance in ovarian cancer via suppression of apoptosis. Gynecologic Oncology, 130(2), 369-376.

Marchini, S., Fruscio, R., Clivio, L., Beltrame, L., Porcu, L., Nerini, I. F., \& D'Incalci, M. (2013). Resistance to platinum-based chemotherapy is associated with epithelial to mesenchymal transition in epithelial ovarian cancer. European Journal of Cancer, 49(2), 520-530.

Marsh, S., McLeod, H., Dolan, E., Shukla, S.J., Rabik, C.A., Gong, L., Hernandez-Boussard, T., Lou, X.J., Klein, T.E., \& Altman, R.B. (2009). Platinum pathway. Pharmacogenet Genomics, 19(7), 563-564.

Nakada, M., Minamoto, T., Pyko, I. V., Hayashi, Y., \& Hamada, J.-I. (2011). The pivotal roles of GSK3 $\beta$ in glioma biology. Molecular Targets of CNS Tumors.

Nestheide, S., Bridge, J. A., Barnes, M., Frayer, R., \& Sumegi, J. (2013). Pharmacologic inhibition of epigenetic modification reveals targets of aberrant promoter methylation in ewing sarcoma. Pediatric Blood \& Cancer, 60(9), 1437-1446.

Qin, T., Si, J., Raynal, N. J.-M., Wang, X., Gharibyan, V., Ahmed, S., \& Issa, J.-P. J. (2015). Epigenetic synergy between decitabine and platinum derivatives. Clinical Epigenetics, 7(1), 97.

Tang, J., Fang, F., Miller, D. F., Pilrose, J. M., Matei, D., Huang, T. H.-M., \& Nephew, K. P. (2014). Global DNA methylation profiling technologies and the ovarian cancer methylome. Cancer Epigenetics, 1238, 653-675.

Thorn, C. F., Oshiro, C., Marsh, S., Hernandez-Boussard, T., McLeod, H., Klein, T. E., \& Altman, R. B. (2011). Doxorubicin pathways: pharmacodynamics and adverse effects. Pharmacogenetics and Genomics, 21(7), 440-446.

Vybrant ${ }^{\circledR}$ MTT cell assay kit (V-13154). (2002). Retrieved from ThermoFisher website: https://tools.thermofisher.com/content/sfs/manuals/mp13154.pdf Zhang, X., Wang, X., Song, X., Liu, C., Shi, Y., Wang, Y., \& Zhang, L. (2010). Programmed cell death 4 enhances chemosensitivity of ovarian cancer cells by activating death receptor pathway in vitro and in vivo. Cancer Science, 101(10), 2163-2170.

Zeller, C., Dai, W., Steele, N. L., Siddiq, A., Walley, A. J., WilhelmBenartzi, C. S., \& Brown, R. (2012). Candidate DNA methylation drivers of acquired cisplatin resistance in ovarian cancer identified by methylome and expression profiling. Oncogene, 31(42), 4567-4576. 